\title{
Ahab's Leg: Exploring the Issues of Communicating Semi-formal Requirements to the Final Users
}

\author{
Chiara Leonardi, Luca Sabatucci, Angelo Susi, and Massimo Zancanaro \\ Fondazione Bruno Kessler - IRST CIT \\ Via Sommarive, 18 I-38050 Povo, Trento \\ \{cleonardi, sabatucci, susi, zancana\} @fbk.eu
}

\begin{abstract}
In this paper, we present our experience in using narrative scenarios as a tool to communicate and validate semi-formal requirements with the stakeholders in a large software project. The process of translating the semi-formal language of Tropos into the narrative form of scenarios is introduced and some unintended implications of this process are discussed. In particular, we define the notion of Ahab's leg to describe the necessity to introduce new constraints or features in a description when moving to a different representational language. Starting from the lessons learned with this specific case study, we derive some general implications concerning the issue of requirement translation for validation tasks and we propose some methodological guidelines to address the Ahab's leg dilemma.
\end{abstract}

Keywords: Goal-Oriented Requirements Engineering, User-Centred Design, Persona, Scenario.

\section{Introduction}

The focus group with the stakeholders was proceeding in a satisfactory way when suddenly in discussing a scenario one of the nurses commented negatively about the use of sensors located on the doors. She complained that the doors in their facilities are wider than the one depicted in the scenario and that for security reasons they should never be closed. A very lively discussion began about the possibility of locking the doors in a nursing home while the facilitator tried to focus the attention of the group to the functional requirement to be validated: that is, the need to trigger an alarm if a guest leaves a common room.

Situations like this happen very often when a design team wants to validate late requirements with stakeholders: although narrative scenarios are powerful tools to represent and communicate requirements to non-technical people, it may be the case that stakeholders focus their attention on non-central aspects. This paper discusses our experience in using narration as a tool to communicate and validate semi-formal requirements with the stakeholders of a large software project. We encountered the kind of problems above in different forms when we had to decide how to instantiate the formal concepts in a narrative form and which details have to be added to make the story more engaging for the stakeholders. In this paper, we present the process of 
translating the semi-formal language of Tropos into the narrative form of scenarios and some unintended implications of this process.

The importance of narration for mediating and discussing requirements with stakeholders has already been discussed in requirements engineering (RE) $[1,4,10,13,14,15]$. While several studies addressed the issue of integrating scenarios in the requirements elicitation phase, few works specifically considered the challenge of using scenarios-and in particular scenarios represented in a narrative form-to communicate and validate requirements with stakeholders. Efficient communication and iterative validation of requirements with stakeholders is a key challenge for RE. The issue of adequately communicating and negotiating requirements with stakeholders and software engineers still challenges approaches based on formal representation of requirements.

In the context of a large software project, we used narrative visual scenarios to efficiently communicate requirements collected in the field and to provide all participants-both software engineers and professionals-with adequate information to envision innovative and useful services. The semi-formal methodology Tropos was used to filter information, maintain traceability and provide tools for requirements conflict analysis. This paper addresses the advantages and shortcomings of the complementary use of semi-formal descriptions and narrative informal descriptions for the purposes of requirements validation focusing on the challenges posed by the translation between the two. Starting from the lessons learned within a specific case study, we derive some general implications concerning the issue of requirement translation for validation tasks.

In Section 2 we introduce the conceptualization of the Ahab's Leg dilemma, that is, the necessity to add more constraints or features to a description when moving to a different representational language. Section 3 briefly summarizes the methodology and the techniques used for requirement elicitation and management in our project and the issue we encountered. Section 4 introduces a post-analysis of the scenarios used and a proposal for a methodological framework to limit and manage the impact of Ahab's legs in a validation process.

\section{The Ahab's Leg Dilemma}

In the famous novel Moby Dick, the main character, Captain Ahab, has a peg-leg. The author, Herman Melville, told us that it is made from a whale jaw but nothing is said whether it is the left leg or the right one. In 1956, John Houston directed a film adaptation of the book starring Gregory Peck as Captain Ahab. He and the screenwriter, the novelist Ray Bradbury, were forced, because of the constraint of the visual media, to decide that the left leg was the whale bone peg. Although the peg-leg is a fundamental part of the story (you cannot imagine any adaptation of the book for which Ahab does not have a peg-leg), knowing which one has no bearing on it. Yet, when the peg-leg is instantiated, this decision may generate a lot of consequences, some of them might be harmless and some might not ${ }^{1}$.

\footnotetext{
${ }^{1}$ The Ahab's leg example was introduced by Eco [7] in discussing the problem of translation.
} 
The Ahab's leg dilemma consists of the necessity to add more details to the original storyline, because of the different characteristics of the target media (visual vs. textual, in the case of Moby Dick) or because of the use of a different communication style, dramatization vs. neutral description, that requires the story to be engaging (as in our example above). Ahab's legs are often unavoidable and they do not necessarily represent a problem unless they bring the viewers (or the stakeholders) to draw unwanted inferences that can contradict other aspects of the story or, as in our example above, divert their attention from the important aspects of the story.

In the case of scenarios derived from requirements, Ahab's legs may be introduced because abstract requirements, summarized as short and clear sentences, are translated into full-fledged narrations. In this process, usually information must be added in order to raise the dramatic tension to the story (the importance of engagement in scenarios is well known in literature [8]) and to instantiate requirements in a concrete spatial-temporal context.

It is worth noting that not every problem encountered when validating scenarios during group discussions can be classified as Ahab's legs. Problems may, for example, be related to the group dynamics rising in focus groups that possibly drift the topic of discussion. Actually, focus groups, different from other methods, require greater attention and the role of moderator is crucial in keeping the group discussion on track [12]. In other cases, when stakeholders complain about a specific aspect of a scenario, it may be the case that the corresponding requirement is wrong or not well understood by the designers. Indeed, spotting these problems in the requirements is precisely the purpose of scenarios as we used them.

The problems raised by Ahab's legs do not correspond to any part of an actual requirement and therefore any discussion about them is a useless waste of time. It is worth noting that Ahab's legs do not necessarily induce shortcomings in validating requirements. In many cases stakeholders are able to avoid discussions deemed irrelevant, especially, as noted above, if the focus groups are effectively moderated by a professional facilitator.

The Ahab's legs can thus be recognized as translation challenges. The possibility of such problems is also recognized by authors in the field of RE (see for example [14]). Interesting suggestions are given by Marasco [11] who underlines the shortcomings but at the same time the necessity to create different views of requirements, highlighting the importance of bridging the gap between text-based and visual requirements representation to improve the quality of requirements in terms of completeness and validity. Still, no systematic analysis has been done to understand and provide concrete solutions to help designers and analysts cope with different views of requirements, in particular between semi-formal representation and narration.

\section{Methodology and Techniques}

ACube is a large research project funded by the local government of the Autonomous Province of Trento in Italy with the aim of designing a highly technological smart environment to be deployed in nursing homes as a support to medical and assistance staff. An activity of paramount importance was the analysis of the system requirements for what concern cost containment and quality improvement of services in specialized centers for people with severe motor or cognitive impairments. From a 
technical point of view, the project envisages a network of sensors distributed in the environment or embedded in users' clothes. This technology should allow monitoring the nursing home guests unobtrusively, that is, without influencing their usual daily life activities. Through advanced automatic reasoning algorithms, the data acquired through the sensor network will be used to promptly recognize emergency situations and to prevent possible dangers or threats for the guests themselves.

The ACube project consortium has a multidisciplinary nature, involving software engineers, sociologists and analysts, and is characterized by the presence of professionals representing end users directly engaged in design activities. A User Centered Design (UCD) approach was implemented to manage the multidisciplinary effort of balancing stakeholders' needs and technical constraints. The integration of UCD methods with the goal-oriented requirements engineering methodology Tropos was meant to assure the validity, completeness and traceability of requirements.

In the following we briefly discuss the two methodologies employed in our study and how they were jointly used during the project.

\subsection{Tropos}

The Tropos methodology [3,17] relies on a set of concepts, such as actors, goals, plans, resources, and dependencies to formally represent the knowledge about a domain and the system requirements. An actor represents an entity that has strategic goals and intentionality within the system or the organizational setting. An actor is used to model both human stakeholders and software and hardware systems. Goals represent states of affairs an actor wants to achieve. Executing a plan can be a means to realize a goal. Actors may depend on other actors to attain some goals or resources or for having plans executed. Tropos models are visualized through actor and goal diagrams. The former are graphs whose nodes represent actors and arcs are strategic dependencies between pairs of actors. A goal diagram represents an individual actor perspective in terms of its main goals, and their decomposition into sub-goals. Furthermore, plans and resources that provide means for goal achievement are depicted through means-end relationships.

Tropos distinguishes five phases in the software development process: Early Requirements, where the organizational domain is described, Late Requirements, where the system-to-be is introduced in the organization, System Architecture Design, System Design and System Implementation. In the project, we applied the first two phases of the methodology to describe the nursing homes organizational setting and stakeholders' needs and to investigate the technical requirements for the ACube system.

\subsection{Personas and Visual Scenarios}

Usually, in the practice of requirements engineering, scenarios have been intended mainly as abstract descriptions of systems functionalities. In this project, we took a slightly different stance by employing narrative scenarios and personas in the way they are used within the field of Interaction Design (ID) [6]. Narrative scenarios are stories characterized by their brevity and simplicity which represent people acting in a specific context and supported by technologies. Scenarios make concrete the behavior of a service as experienced by specific, though fictional, users. They help design 
teams in negotiating a shared representation of the domain and hence a more effective elicitation of requirements. In ID, scenarios are proposed to be used in several phases of the design, from early requirement elicitation to design validation. Actually, as recently stressed by Katasonov et al. [9], a major problem in requirement quality control is the achievement of a satisfactory level of understanding on the requirements by stakeholders especially when they lack technical expertise and do not share the same (formal and abstract) language of analysts and engineers. Due to the assumption that validating requirements is more an issue of efficiently communicating and iteratively negotiating knowledge than a linear process of checking a given corpus of data, in our study we designed visual scenarios as communication tools to allow technical and non-technical partners to symmetrically contribute to requirements validation and refinement. We adopted the specific scenarios approach as developed by Carroll and Rosson [4] and subsequently enriched by Cooper with the notion of personas [5], Personas are rich descriptions of archetype users meant to draw attention on users' goals and motivations. Introducing personas in scenarios-based approach provides an anchor against self-referentiality in design and makes scenarios more concrete. Personas (an example of personas is given later in Section 3.3) are created starting from data gathered from actual users interviewed and observed through contextual inquiries. They are usually evaluated with respect to their believability for the stakeholders before creating the actual scenarios. It is worth noting the difference between Tropos actors and personas. While actors are abstract entities and they are not sufficiently concrete to provide understanding of empathy with users, personas are expected to engage the empathy that helps the designers, stakeholders and software engineers to make decisions on both the cognitive and emotional sides.

Table 1. Main characteristic of TROPOS and ID methods

\section{TROPOS}

- Exhaustive picture of the domain.

- Abstract representation of the domain.

- Static and invariant picture of the domain.

- Do not provide specific tools for finer prioritizing requirements than the reasoning on alternatives and contributions.

- Neutral representation that do not engender an emotional response.

- Do not provide information about the physical context.

- Provides a general representation of invariant dependencies among actors.

- Support traceability.

\section{PERSONASISCENARIOS}

- Selection of specific situations that are described in a narrative form (coverage problem).

- Concrete representation of the domain.

- Dynamic representation involving the spatiotemporal dimension.

- Stories provide a support for prioritizing requirements.

- "Dramatic" representation that engenders empathy.

- Provide details about the physical context in which people act.

- Provide details about how interactions occur in a given specific situation.

- Do not support traceability.

\subsection{Joint Process: Concurrent Use of Tropos and ID}

In ACube, we made joint use of the semi-formal Tropos language and ID methods throughout the whole project lifecycle, in particular from early requirements identification to final validation with stakeholders. 
The two approaches explore different dimensions of the domain and of the design space of the system using complementary approaches, tools and languages (see Table 1). In this context, one of the most relevant problems is the perspective and foucs of the two research approaches: ID methods strongly emphasize users and the contexts in which they behave while Tropos focuses on roles and goals, promoting a more abstract level of description that is typical in software engineering techniques. In order to effectively integrate the different methods we developed a framework to allow for the alignment of the approaches, their languages and to facilitate a shared representation of data. Table 2 illustrates the four phases that caracterized the process: Early exploration, Problem identification, Envisioning, and Validation. For each phase of the process the table shows the contributions of the two methodologies and artifacts that allowed to maintain an alignement between the two representations.

Table 2. The four phases that characterized the process and artifacts used for communication/integration issues

\begin{tabular}{|c|c|c|c|c|}
\hline $\begin{array}{l}\text { Phases/ } \\
\text { methods }\end{array}$ & $\begin{array}{l}\text { 1. Early explo- } \\
\text { ration }\end{array}$ & $\begin{array}{l}\text { 2. Problem } \\
\text { identification }\end{array}$ & 3. Envisioning & 4. Validation \\
\hline $\begin{array}{l}\text { UCD } \\
\text { methods }\end{array}$ & Contextual inquiries & $\begin{array}{l}\text { Definition of } \\
\text { personas }\end{array}$ & $\begin{array}{l}\text { Participative work- } \\
\text { shops to develop } \\
\text { envisioning scenar- } \\
\text { ios }\end{array}$ & $\begin{array}{l}\text { Scenarios discus- } \\
\text { sion with the } \\
\text { stakeholders }\end{array}$ \\
\hline \multirow{2}{*}{$\begin{array}{l}\text { Communi- } \\
\text { cation/ } \\
\text { integration } \\
\text { tools }\end{array}$} & $\begin{array}{l}\text { Descriptive table } \\
\text { (narrative) }\end{array}$ & Personas & $\begin{array}{l}\text { Narrative techno- } \\
\text { logical scenarios } \\
\text { and storyboards }\end{array}$ & $\begin{array}{l}\text { Narrative descrip- } \\
\text { tion and story- } \\
\text { boards }\end{array}$ \\
\hline & $\begin{array}{l}\text { Actor-Action- } \\
\text { Resource-Goal } \\
\text { Analysis }\end{array}$ & Critical Aspects & $\begin{array}{l}\text { Positive-negative } \\
\text { contributions }\end{array}$ & $\begin{array}{l}\text { Functional and } \\
\text { Non-functional } \\
\text { Requirements }\end{array}$ \\
\hline $\begin{array}{l}\text { Tropos } \\
\text { methods }\end{array}$ & $\begin{array}{l}\text { Domain knowledge, } \\
\text { Early actor model- } \\
\text { ing }\end{array}$ & $\begin{array}{l}\text { Early Requirements } \\
\text { Phase }\end{array}$ & $\begin{array}{l}\text { Late Requirement } \\
\text { Phase }\end{array}$ & $\begin{array}{l}\text { Requirements } \\
\text { Refinement }\end{array}$ \\
\hline
\end{tabular}

Table 3. Some of the personas used in ACUBE Project

\begin{tabular}{|l|l|}
\hline $\begin{array}{c}\text { Sabrina Age: } 40 \\
\text { Health operator, she assists hosts in daily } \\
\text { activities. Her activities: (i) monitor } \\
\text { dangerous events, analysis of the kind of } \\
\text { event, (ii) raise up an alarm via phone or } \\
\text { direct contact with operators, (iii) moni- } \\
\text { tor patient conditions. }\end{array}$ & $\begin{array}{l}\text { Piera } \\
\text { She's lived in a Nursing Home for 6 years. She is not } \\
\text { self-sufficient because of health and motion disabilities. } \\
\text { She likes the human side of her work but } \\
\text { frequent anxiety and agitation. She wishes for more } \\
\text { human relationships with caregivers, nurses and rela- } \\
\text { tives. }\end{array}$ \\
\cline { 2 - 2 } $\begin{array}{l}\text { bureaucratic matters. Problems: hard to } \\
\text { follow the hosts in absence of an ade- } \\
\text { quate number of health workers. }\end{array}$ & $\begin{array}{l}\text { Maria } \\
\text { She's lived in a Nursing Home for 1 year. She suffers } \\
\text { from Alzheimer's at middle level with lack of memory } \\
\text { and confusion. She tried to leave the institute to go back } \\
\text { home once, thus caregivers look at her movements with } \\
\text { a special attention. }\end{array}$ \\
\hline
\end{tabular}


1. Early exploration. The process started with the investigation of the domain to understand the organizational setting in representative sites and to derive possible needs and a set of possible services that the system may provide to users. In the ACube project, contextual interviews [2] were performed in 4 different nursing homes and involved about 40 health professionals including health workers, nurses, medical staff and managers. Information retrieved via contextual inquiry are represented via Tropos actor-goal early requirement models.

2. Problem setting. The analysis of critical aspects has been developed to highlight the main problems that professionals of nursing homes experience in their job. We defined a set of personas (see Table 3 ) and generated narrative descriptions of these personas deploying their daily working activities in a specific context, using resources to reach their goals. The aim was to represent criticalities that may be addressed through a technological intervention. Here, Tropos models support the representation of critical aspects via the characteristics of actors and goals.

3. Envisioning. A participative workshop has been organized to analyze how technology could positively intervene in activities thus supporting the achievement of goals and resolving critical issues identified in the problem identification phase. It is worth noting, that in this phase our main goal was investigating high level requirements and not producing design ideas. About 10 participants attended the workshop including the ID team and representatives of stakeholders and technologists. The heterogeneity of the group was meant to guarantee the generation of creative but feasible ideas, to provide concrete solutions to problems identified by nursing home professionals as well as to provide solutions that could meet engineers' expectations and their research interests. Outcomes were pursued at multiple levels: to expand the designer's perspective and to see the problems from different points of view, to figure out how their ideas can work in a real context, to identify design criticalities and open issues, to generate requirements of the system-to-be. The workshop ended with the definition of 5 different macro-services the ACube system might provide.

As a consequence of the envisioning focus group, and the introduction of the system into the organization, the Tropos process moved from the early requirement phase to the late requirement phase. Figure 1 shows an excerpt of the Tropos model, describing a small part of the goals and the activities of the SeniorOSS actor (a caregiver in the nursing home). In particular the actor Senior OSS has the goal to [prevent dangerous behavior in patients] that can be AND decomposed in [monitor patients in her visual area] and [coordinate interventions in the nursing home area].

This latter goal is delegated to the ACube System actor via the goal delegations [identify a guest dismissing the group] and [receive alerts of relevant events]. These goals are two requirements to be satisfied by the system that must instantiate them (means-ends relationships) via the plans [monitor patients] and [send alarms] respectively.

4. Validation. Two focus groups were organized with stakeholders and the technical staff for validating the list of requirements produced in the previous phase. Due the importance of this step for the topic of the paper, this is part is discussed in detail in the following subsection. 


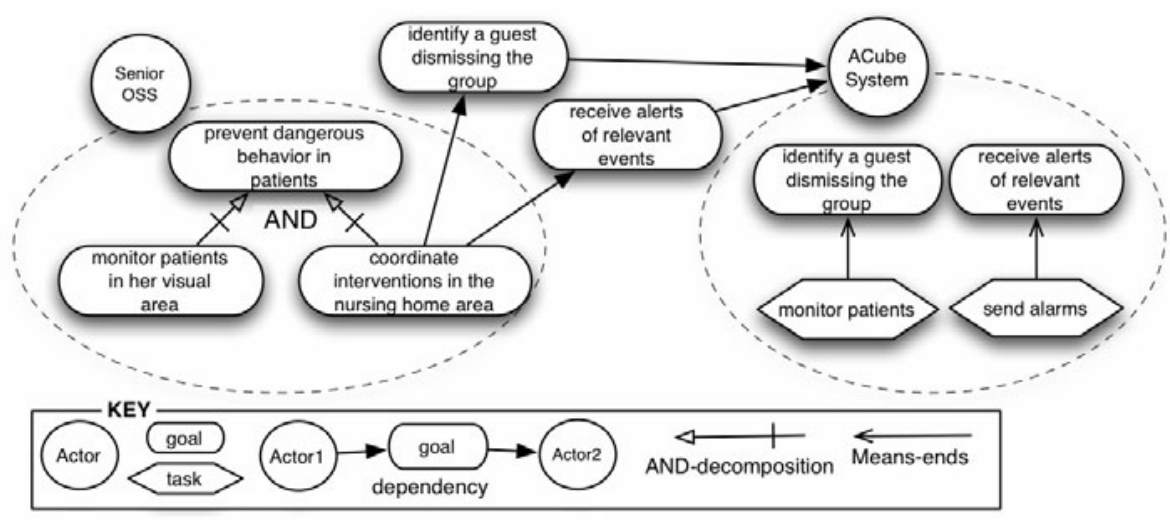

Fig. 1. An excerpt of the Tropos model for the nursing home

\subsection{The Validation Phase}

After the preparation of the Tropos late requirement diagrams, and the corresponding list of requirements, we started the validation phase. Simple visual scenarios were designed to make the list of requirements more understandable by partners. To generate scenarios we imagined how the system could support personas to cope with problematic situations during their daily work. Macro-services emerged in the envisioning phase have been instantiated into concrete - but non exhaustive - representations of the system functionalities. Eventually, 5 visual scenarios were generated, each addressing a problematic situation identified in nursing homes accompanied by one of the possible technological solutions.

A first focus group was held with the representatives of the 10 research groups involved in ACube project, 27 people attended the meeting. The second focus group was organized with the stakeholders, 3 managers of nursing homes previously involved in the early exploration phase attended the meeting. The goal of these meetings was to assess of the validity, acceptability and feasibility of requirements and to envision alternatives not considered in the scenarios.

The structure of the two meetings was the following: first, a general presentation was given to introduce the goals of the meeting and to discuss general results collected in the fieldwork. Then, for each of the five scenarios generated we introduced: the context of the scenario - organization context and personas acting in those contexts, the rationale for the scenario, which is the criticality we wanted to address with that specific scenario. Subsequently, the scenarios were represented in a visual form through storyboards (see Figure 2). Finally, criticalities the scenarios could rise - in terms of technological feasibility and acceptability for end-users - and the underlying abstract requirements that the scenarios instantiate were presented to trigger the discussion. For each scenario, 20 minutes of discussion followed. A moderator was in charge of driving the discussion on the specific dimensions we wanted to assess.

The workshop with technological partners was focused on technical feasibility and research interest, and on the envisioning of original solutions to the critical situations identified. Acceptability and usefulness were instead the pivots of the workshop with 


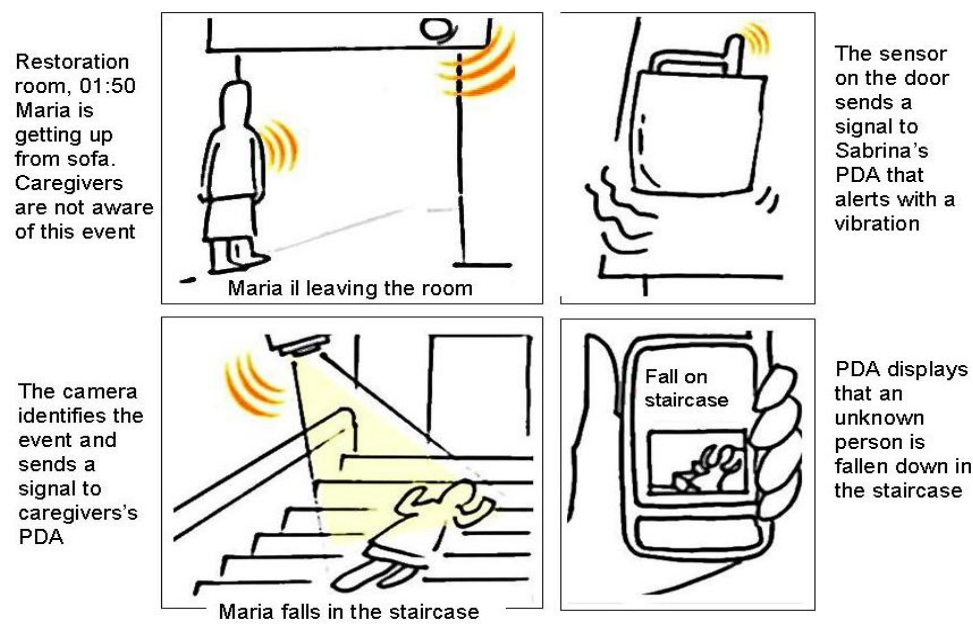

Fig. 2. A scenario extracted from the Tropos model of the domain

end-users representatives. Expected outcomes of the two focus groups were the emergence of design criticalities, the resolution of open problems and the identification of new propositions and ideas, in order to collect additional elements to elaborate an organic description of the technological architecture. The role of the moderator was also to focus participants' attention on specific aspects of the scenarios (those directly related to requirements) and to cut discussions concerning non relevant aspects. The output of this phase was the agreement on certain requirements and the refinement of Tropos late requirement diagrams.

Besides the general positive output and the satisfaction of the partners - principally satisfied of the rich discussion emerged around scenarios - several secondary (collateral) issues emerged. Several times the discussion of participants focused on aspects non-relevant for technological purposes. Beside traditional shortcomings usually found when conducting focus groups [12], we identified other impasses pertaining to the kind of information communicated through visual scenarios, leading participants to lose repeatedly the focus of attention. As discussed above, we defined as Ahab's Legs the translation and communication shortcomings rising from the necessity to translate information from one media to another.

\section{Re-thinking the Approach}

The issues we experienced during the focus groups with technological partners and with stakeholders were mainly due to Ahab's legs (AL). This section reports the analysis we conducted, after the validation phase, in comparing the knowledge expressed in the requirements documents with the knowledge mediated by the visual scenarios. 


\subsection{Ahab's Leg Classification}

In order to identify the ALs in our 5 scenarios we compared each frame of each scenario with the corresponding requirement. For example, Table 4 illustrates the case of the requirement [The system identifies when a guest is dismissing the group]. This requirement has been visually represented in the scenario for the emergency monitoring and prevention of falls. This scenario shows a guest of the institute that wears a sensor that sends a signal. This is captured by a receiver that is placed near the door of the room, where the guest is passing through.

This simple piece of scenario includes four ALs: 1) the definition of the time and place in which the scene is set: a wide common room with one door; 2) the couple wearing sensor/receiver is an AL cause it is not yet defined by design how to track guest movements; 3) the receiver placed on the door is another AL, because it refers to a decision to set the device in a precise position of the environment; and 4) the use of arcs going out from the sensor to the receiver is a graphical means for communicating the presence of an interaction in a symbolic way.

With this procedure, we collected 34 ALs from the 28 frames of the 5 scenarios. An analysis based on similarities of occurrences results in 6 different categories of AL. Three of these categories relate to the cause of the difference in translation: (i) the need to introduce a design feature to visualize a part of the system, (ii) the need to make concrete an abstract representation and (iii) the use of symbols that may be misleading. The other three categories related to the impact that the AL has on the target language: (i) the resource used, (ii) the context in which the scene takes place and (iii) the working practice that is represented. These categories are not exclusive and an AL can be classified as belonging to one or more categories.

An AL is generated by an early design when the scenario is constrained to show some design solutions that have not been explicitly chosen but that have been represented in order to elicit underlying problems and suggest concrete solutions. For instance the use of a wearable sensor that sends signals does not come out from the requirements list, but the design team decided to make concrete the presence of the system in such a way. Nevertheless, the presented technical solution is only a possible alternative among others; other solutions could be designed, for instance, the use of cameras spread in the environment was going to be evaluated too. Starting from abstract requirements (such as "The system alerts caregivers of relevant events"), one of the possible design solutions has been visualized ("a PDA in the caregiver's pocket vibrates to signal the event") to communicate to stakeholders one of the possible design solution that could meet that requirement.

An AL can be generated by the level of details due to the specific media used for representing the scene. The typical example is the one that inspired the name of Ahab's leg: the cinematographic version of the novel requires a decision about which is the peg-leg. An example from the project concerns the decision to set the scene in a specific kind of environment, a common room, close to a door.

This kind of AL can be minimized by carefully translating abstract information to a concrete scene. While a certain contextualization is necessary to create a credible story, the designer should however pay attention to convey only the necessary information, and to maintain abstract those contents that could generate a discussion on non relevant details. In our case it was important to communicate the information that 
the scene was taking place in a common room but we decided to keep all the other information on the environment implicit in order to let stakeholder focus only on the relevant event, such as common room with several patients and few health professional available.

An AL is generated by the use of symbols, typical of comics, that communicates something abstract in a scene, as well as an interaction, a mood, the act of thinking and so on. The twofold risk is to use a symbol whose meaning is not commonly recognized by people, or to communicate in a concrete way something that would be better to maintain abstract. An example of AL in this category is the use of arcs for representing a wireless communication or signal connecting the sensor with the receiver. This graphical expedient is used to show an interaction between two devices, but the risk is stakeholder focus on the direction of the communication (who is the transmitter, who is the receiver). This problem pertains to a more general issue well known within the semiotic research area, that is, inter-semiotic translation, occurring every time a linguistic sign is translated by means of non-linguistic signs (visual or audio texts).

Table 4. Requirements, scenarios and Ahab's legs in our example

\begin{tabular}{|l|l|l|}
\hline \multicolumn{1}{|c|}{ Requirement } & \multicolumn{1}{|c|}{ Scenario Frame } & \multicolumn{1}{c|}{ Ahab's Leg } \\
\hline $\begin{array}{l}\text { 1. The system } \\
\text { identifies when a } \\
\text { guest is dismissing } \\
\text { the group }\end{array}$ & $\begin{array}{l}\text { 3.00 pm, common room. The scene } \\
\text { shows a guest of the institute that } \\
\text { wears a sensor that sends a signal. } \\
\text { This is captured by a receiver that is } \\
\text { placed near the door of the room, } \\
\text { where the guest is passing through }\end{array}$ & $\begin{array}{l}\text { AL1: resources - wearable } \\
\text { sensor and receiver } \\
\text { AL3: receiver on the door }\end{array}$ \\
\hline $\begin{array}{l}\text { 2. The system alerts } \\
\text { caregivers of rele- } \\
\text { vant events }\end{array}$ & $\begin{array}{l}\text { The scene shows (and describes) a } \\
\text { PDA in the caregiver's pocket that } \\
\text { vibrates }\end{array}$ & $\begin{array}{l}\text { AL4: resource - PDA } \\
\text { AL5: caregivers have a de- } \\
\text { vice in their pocket } \\
\text { AL6: vibration for alerting } \\
\text { the caregiver }\end{array}$ \\
\hline
\end{tabular}

Among impact we identify: (i) resource, (ii) context and (iii) working practices. An AL may influence a resource (typically a technological device) that the system will introduce in the environment, or already existing in the domain. An example is the introduction of a sensor in the environment that tracks guest movements. An AL may influence the context represented in a scenario, adding details about the time (for instance by specifying when the scene is set: 'at 3:00 pm'), about the space (for instance by specifying where the scene is set: 'in the common room'), about a condition or an event that is occurring (for instance 'the guest is moving through the door') or a quantity (for instance specifying how many guest and caregivers are present). An AL may also add details about a working practice or a methodology that caregivers will adopt as a consequence of the system-to-be. The scene provides details (for instance about decisions that are taken or activities that are executed) just because the dramatization of the story needs a plot in which personas act for solving emergencies. 
This classification has been developed starting from a specific case and it has not yet been completely investigated. Therefore, we cannot at this stage claim that is of general purpose. However this classification is coherent with the categories identified by Eco [7]. Table 5 reports a subset of the ALs we identified in this project.

This analysis has been used to provide a rationale to each AL found in the 5 scenarios and to decide whether it could have been removed or not. The scenarioauthoring activity should be iterated by considering the evidence of each AL and considering the relevance of the corresponding detail in the scene. If the detail can be removed without losing important data that designers want to communicate, the scenario should be redefined. For example, in the case of the AL3 [the receiver placed on the door], this detail could have easily been removed reducing the risk to focus stakeholders' attention to technological details that were not yet discussed.

Table 5. Classification of the Ahab's Legs in our example

\begin{tabular}{|l|c|c|c|c|c|c|}
\hline & $\begin{array}{l}\text { Early } \\
\text { Design }\end{array}$ & $\begin{array}{c}\text { Level of } \\
\text { Details }\end{array}$ & $\begin{array}{l}\text { Use of } \\
\text { Symbols }\end{array}$ & Resource & Context & $\begin{array}{l}\text { Working } \\
\text { Practice }\end{array}$ \\
\hline AL1: time and place & & $\sqrt{ }$ & & & $\sqrt{ }$ & \\
\hline $\begin{array}{l}\text { AL2: wearable sensor and } \\
\text { receiver }\end{array}$ & $\sqrt{ }$ & & $\sqrt{ }$ & $\sqrt{ }$ & & \\
\hline AL3: receiver on the door & & $\sqrt{ }$ & & & $\sqrt{ }$ & \\
\hline AL4: resource - PDA & $\sqrt{ }$ & & $\sqrt{ }$ & & & \\
\hline $\begin{array}{l}\text { AL5: caregivers have a } \\
\text { device in their pocket }\end{array}$ & & $\sqrt{ }$ & & & & $\sqrt{ }$ \\
\hline $\begin{array}{l}\text { AL6: vibration for alerting } \\
\text { the caregiver }\end{array}$ & $\sqrt{ }$ & & $\sqrt{ }$ & & & \\
\hline
\end{tabular}

The categorization of the AL dilemma brought us to propose some guidelines to systematically approach the elimination of some irrelevant details from scenarios. In the cases in which removing an AL is not possible or too complicated, it is very important to frame the scenario (for example with an introductory description) in such a way that the discussion from the AL is averted as much as possible.

\subsection{Toward a Methodology for Translating Requirements into Scenarios}

When we prepared our narrative scenarios we were aware of the possible communication problems that may occur during the validation phase, thus we spent a lot of effort in preparing the meeting, to direct the conversation in the desired direction. Despite this preliminary work, we have been unable to avoid having stakeholders sometimes concentrate on some secondary aspects of the narration (for example the discussion about the doors of the room in the introduction). The post-analysis conducted on the scenario before the validation experience revealed a bigger number of ALs than we recognized at the beginning. This suggested to introduce a scenario-refinement activity in our analysis process in order to consider whether the use of each AL was really beneficial to the scenario (because it supported a greater level of engagement or made a requirement clearly visible, for example) or was just a distracting narrative element. 


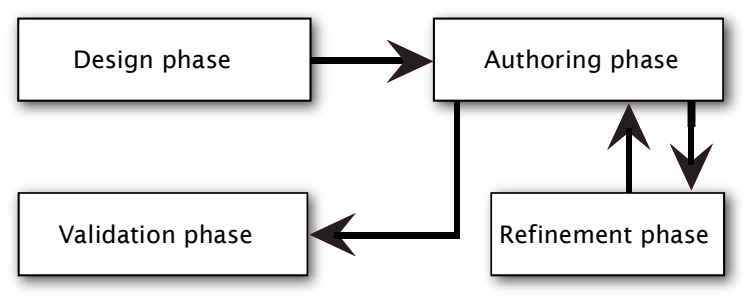

Fig. 3. A methodology for translating requirements into scenarios

The methodology for moving from requirements to their validation with stakholder can be summarized as (see Figure 3): a design phase in which requirements are draft, a scenario authoring phase in which these requirements are represented in a narrative (and often visual) format. Finally, the validation meeting in which scenarios are presented and discussed with stakeholders. We propose to introduce a refinement phase after the scenario authoring and before the validation meeting. For each scenario an AL detection activity is executed, that may lead to re-elaborate the scenario representation in order to reduce the number of irrelevant details. The steps can be summarized as follows. (i) Sort requirements by the scenario in which they are represented; (ii) divide the scenario in frames, each is a self-explicatory part of the story; (iii) compare the requirement with the frame and record each detail that is added for enriching the story as an AL; (iv) use the classification schema for evaluating the importance of the presence of the AL to the aim of telling the story; (v) proceed to the elimination of the surplus details from the story or to their modification. It is worth noting that scenarios and not requirements are subject to changes during the refinement phase.

As we already mentioned removing ALs from a narrative description is by no means the only way to manage ALs. For example, when a fieldwork analysis was conducted before the requirement phase, the illustrative material (photos, sketches, interviews, etc.) may be used to add details to the narrative scenarios that result "natural" and therefore less distractive for the stakeholders. Finally, the remaining ALs may be the properly framed by the facilitator to reduce the risk to discuss them for too long.

\section{Conclusion}

In this paper, we discussed some issues that emerged in a large research project when we tried to validate our requirements with the stakeholders by using narrative scenarios instead of the textual version of the requirements themselves. This practice is sometimes used in RE, nevertheless several authors acknowledged the problem of running into misunderstanding when minor details are added to the narration to enrich it and make the presentation concrete and easy to understand. We identified this translation challenge as the Ahab's leg problem.

Although only a few ALs creates problems during validation with stakeholders, several more were identified in a post-analysis of the scenarios generated. Six nonexclusive categories of ALs have been identified by the analysis. Consequently we 
propose a methodology to manage the insurgence of ALs that consists in a scenariorefinement phase in which every AL is checked and, when possible, is eliminated or modified in order to be understandable by stakeholder without conveying undesired meanings. It is worth noting that to some extent ALs may also be considered beneficial. In a ID perspective, showing a hypothetical vision of the system-to-be is a way to open up the discussion on design in order to redefine the problem with stakeholders. In this respect, ALs may help to foster the discussion on some not-central but still very relevant dimensions of the problem. This approach is recommended by a modern approach to ID [19]. Yet, even in these cases, ALs should still to be carefully managed by clearly communicating the goals and motivations behind their introduction.

Finally, the problem of generalization is still an open question on our work. By comparing them with categories identified in semiotics studies, we assume that they are general enough to be applied in other contexts but more data is needed for assessing the classification. Another aspect that still needs attention is how to relate the AL categories identified in the post analysis to systematic procedures for controlling (limiting or encouraging) the occurrence of ALs in the scenario specification.

\section{Acknowledgments}

The research was funded by the Autonomous Province of Trento, project ACube (Grandi Progetti 2006).

\section{References}

1. Aoyama, M.: Persona-and-Scenario Based Requirements Engineering for Software Embedded in Digital Consumer Products. In: Proc. of 13th IEEE International Requirements Engineering Conference (RE 2005), pp. 85-94 (2005)

2. Beyer, H., Holtzblatt, K.: Contextual Design: Defining Customer-Centered Systems. Morgan Kaufmann, San Francisco (1998)

3. Bresciani, P., Giorgini, P., Giunchiglia, F., Mylopoulos, J., Perini, A.: Tropos: An AgentOriented Software Development Methodology. Autonomous Agents and Multi-Agent Systems 8(3), 203-236 (2004)

4. Carroll, J.M., Rosson, M.B.: Getting around the task-artifact cycle: How to make claims and design by scenario. ACM Transaction on Information Systems 10, 181-212 (1992)

5. Cooper, A.: The Inmates are Running the Asylum. SAMS Publishing, USA (1991)

6. Cooper, A., Reimann, R., Cronin, D.: About Face 3: The Essential of Interaction Design. Wiley Publishing, Chichester (2007)

7. Eco, U.: Kant and the platypus: essays on language and cognition. Harvest Books (2000)

8. Grudin, J., Pruitt, J.: Personas, participatory design and product development: an infrastructure for engagement. In: Proceedings of Participatory Design Conference 2002 (June 2002)

9. Katasonov, A., Sakkinen, M.: Requirements Quality Control: a Unifying Framework. Requirements Engineering Journal 11(1) (2006)

10. Liu, L., Yu, E.: Designing Information Systems in Social Context: A Goal and Scenario Modelling Approach Information Systems 29(2), 187-203 (April 2004) 
11. Marasco, J.: The requirements translation challenge, http: / /articles.techrepublic.com.com/ 5100-10878_11-6128696.html

12. Morgan, D.: Focus groups as qualitative research. Sage Publications, Thousand Oaks (1997)

13. Pohl, K., Haumer, P.: Modelling Contextual Information about Scenarios. In: Proceedings of the Third International Workshop on Requirements Engineering: Foundations of Software Quality REFSQ 1997 (1997)

14. Potts, C., Takahashi, K., Antòn, A.: Inquiry-Based Requirements Analysis. IEEE Software archive 11(2), 21-32 (1994)

15. Rolland, C., Achour, C.B., Cauvet, C., Ralyté, J., Sutcliffe, A., Maiden, N., Jarke, M., Haumer, P., Pohl, K., Dubois, E., Heymans, P.: A proposal for a scenario classification framework. Requir. Eng. 3(1), 23-47 (1998)

16. Seyff, N., Maiden, N., Karlsen, K., Lockerbie, J., Grünbacher, P., Graf, F., Ncube, C.: Exploring how to use scenarios to discover requirements. Requir. Eng. 14(2), 91-111 (2009)

17. Susi, A., Perini, A., Giorgini, P., Mylopoulos, J.: The Tropos Metamodel and its Use. Informatica 29(4), 401-408 (2005)

18. Uchitel, S., Chatley, R., Kramer, J., Magee, J.: Goal and scenario validation: a fluent combination. Requir. Eng. 11, 123-137 (2006)

19. Wolf, T.V., Rode, J.A., Sussman, J., Kellogg, W.A.: Dispelling "design" as the black art of CHI. In: Proceedings of CHI 2006 (2006) 